EPJ Web of Conferences 60, 20007 (2013)

DOI: $10.1051 /$ epjconf $/ 20136020007$

(C) Owned by the authors, published by EDP Sciences, 2013

\title{
Heavy Flavor production with ATLAS
}

\author{
Constantinos Melachrinos ${ }^{1, a}$, for the ATLAS collaboration \\ ${ }^{1}$ University of Chicago
}

\begin{abstract}
ATLAS has a rich heavy flavor and quarkonia program with measurements and searches that can contribute to a better understanding of Quantum Chromodynamics. Using data from proton-proton collisions in the LHC at $\sqrt{s}=7 \mathrm{TeV}$ the $\Upsilon(1 \mathrm{~S}, 2 \mathrm{~S}, 3 \mathrm{~S})$ production cross sections and the charged $B$ meson production cross section measurements are presented, along with comparisons with theoretical expectations. The observation of $J / \psi$ produced in association with $W$ bosons, accessible for the first time, can provide new insights into the hadro-production of charmonium.
\end{abstract}

\section{Introduction}

Quantum Chromodynamics (QCD) describes the strong force that binds quarks together via the mediation of gluons. The study of the production of $\Upsilon$ mesons and $B$ hadrons at the Large Hadron Collider (LHC) allows tests of QCD calculations at a new energy regime, in wider range of rapidity $(y)$ and transverse momentum $\left(p_{T}\right)$ than previously possible at hadron colliders. ATLAS measures the production rate of $\Upsilon$ mesons and $B$ hadrons in the central rapidity region and can extend to high transverse momentum $(\approx 100 \mathrm{GeV})$. The associated production of a $W$ boson with a $J / \psi$ provides an independent probe of the charmonium production mechanism at hadron colliders, and is also sensitive to multiple parton interactions in the colliding protons. In these proceedings, we present the most recent ATLAS results on $\Upsilon$ production [2], charged $B$ production [3] and $W+J / \psi$ associated production [4].

\section{The ATLAS detector}

ATLAS is a multi-purpose LHC detector ${ }^{1}$ designed to study a variety of phenomena [1] . The inner detector (ID) performs tracking of charged particles for $|\eta|<2.5$, surrounded by a superconducting solenoid with a $2 \mathrm{~T}$ magnetic field. The calorimeter system covers $|\eta|<4.9$ and detects energy deposits. The muon spectrometer (MS), outside the calorimeters, measures muon momenta using aircore superconducting toroidal magnets. A typical muon will traverse three precision position measurement stations

\footnotetext{
a e-mail: constantinos.melachrinos@ cern.ch

${ }^{1}$ ATLAS [1] uses a right-handed coordinate system with its origin at the nominal interaction point (IP) in the centre of the detector and the $z$-axis along the beam pipe. The $x$-axis points from the IP to the centre of the LHC ring, and the $y$-axis points upward. Cylindrical coordinates $(r, \phi)$ are used in the transverse plane, $\phi$ being the azimuthal angle around the beam pipe. The pseudorapidity is defined in terms of the polar angle $\theta$ as $\eta=-\ln \tan (\theta / 2)$. The distance $\Delta R$ in $\eta-\phi$ space is defined as $\Delta R=\sqrt{\Delta \eta^{2}+\Delta \phi^{2}}$
}

for $|\eta|<2.7$. Fast trigger chambers cover $|\eta|<2.4$. The MS has a cylindrical barrel geometry for $|\eta| \lesssim 1$ and an endcap disk geometry for $|\eta| \gtrsim 1$. The combination of ID and MS tracking reconstructs muons with $p_{T} \gtrsim 2.5$ $\mathrm{GeV}$ with resolution $\sigma\left(p_{T}\right) / p_{T}$ better than $3 \%$ in the momentum range of interest. Dedicated triggers select events with two muons with invariant mass near the $J / \psi$ and $\Upsilon$ mass, while single high transverse momentum muon triggers accept events that might contain a $W$ or a $Z$ boson.

\section{$3 \Upsilon$ meson production}

The $\Upsilon$ meson is a bound state of a bottom and an antibottom quark. Studying the production cross sections of $\Upsilon$ at the LHC provides information at higher transverse momenta and with better precision due to the large statistics. This may provide new insights into the underlying production mechanisms, which do not yet have a consistent theoretical description that agrees with all experimental observations. The relative production ratios between the different $\Upsilon$ states (1S, $2 \mathrm{~S}, 3 \mathrm{~S}$ ) is also reported.

Using $1.8 \mathrm{fb}^{-1}$ of data, candidate events are preselected using a dimuon trigger with invariant mass in the region of interest (8-11.5 GeV). Muon candidates are required to be reconstructed offline and be within $\Delta R<0.01$ of the corresponding trigger signature. Each muon is required to have $p_{T}>4 \mathrm{GeV},|\eta|<2.3$ and must successfully fit to a common vertex. The dimuon candidates are divided in two regions in rapidity, central $\left(\left|y^{\mu \mu}\right|<1.2\right)$ and forward $\left(1.2<\left|y^{\mu \mu}\right|<2.25\right)$, to exploit differences in mass resolution (120 MeV and $214 \mathrm{MeV}$, respectively). The number of dimuon candidates reconstructed is $3.9 \times 10^{6}$ in the central region and $2.3 \times 10^{6}$ in the forward region. Functions describing the three $\Upsilon$ states $(1 \mathrm{~S}, 2 \mathrm{~S}, 3 \mathrm{~S})$ and the combinatorial backgrounds are fitted to the data to extract the number of $\Upsilon$ candidates for each state, as shown in Fig. 1 for the central region. 
The differential $\Upsilon$ cross section for each state is measured using:

$$
\frac{d^{2} \sigma}{d p_{T} d y} \times \mathcal{B}\left(\Upsilon \rightarrow \mu^{+} \mu^{-}\right)=\frac{N_{\Upsilon}}{\int \mathcal{L} d t \times \Delta p_{T} \times \Delta|y|}
$$

Corrections are applied to account for the efficiency to reconstruct the muons, bin migration effects due to the detector resolution, and theoretical acceptance due to the spinalignment profile of the $\Upsilon$.

The differential cross section as a function of $\Upsilon p_{T}$ is measured in the central and forward regions. The measurements are compared with theoretical expectations based on the color singlet model [5] and the Color Evaporation model [6], as shown in Fig. 1 for the $\Upsilon(1 S)$ in the central region. The production ratios $\Upsilon(2 S): \Upsilon(1 S)$ and $\Upsilon(3 S): \Upsilon(1 S)$ are measured as a function of the $\Upsilon p_{T}$, as shown in Fig. 1 for the central region. Following the initial plateau $(0-5 \mathrm{GeV})$ and the subsequent rise of the ratios with increasing $\Upsilon p_{T}(5-30 \mathrm{GeV})$, a plateau appears to be reached for transverse momenta above $30 \mathrm{GeV}$, accessible for the first time with these measurements. This suggests that direct production of $\Upsilon$ dominates over contributions from excited states decaying in this regime. The rapidity dependence of the ratios is flat.

\section{$4 B^{+}$meson production}

Measurements of $b$-hadron production at the LHC provide tests of QCD calculations at higher center-of-mass energies and wider transverse momentum and rapidity ranges than accessible before. The measurement of the $B^{+}$production cross section ${ }^{2}$, where $B^{+}$decays according to $B^{+} \rightarrow J / \psi K^{+} \rightarrow \mu^{+} \mu^{-} K^{+}$, is presented.

Using $2.4 \mathrm{fb}^{-1}$ of data, candidate events are selected by a di-muon trigger which requires two oppositely charged muons with $p_{T}$ greater than $4 \mathrm{GeV}$ and $|\eta|$ smaller than 2.4, and di-muon invariant mass within $2.5 \mathrm{GeV}<m_{\mu \mu}<$ $4.3 \mathrm{GeV}$. Offline requirements are applied on the reconstructed muons ( $p_{T}>4 \mathrm{GeV}$ and $|\eta|<2.3$ ), as well as the reconstructed $J / \psi$ candidate $\left(2.7 \mathrm{GeV}<m_{\mu \mu}<3.5 \mathrm{GeV}\right)$. The trigger muons and the $J / \psi$ muons are required to be close in $\eta-\phi$ space. The muon tracks of the $J / \psi$ candidates are fitted to a common vertex with an additional third track with $p_{T}$ greater than $1 \mathrm{GeV}$, which is assigned the $K^{ \pm}$mass. To reject background events, the quality of the fit measured by the $\chi^{2}$ per degree of freedom is required to be smaller than 6. $B^{ \pm}$candidates with $p_{T}>9 \mathrm{GeV}$ and $|y|<2.3$ in the mass range $5.04-5.80 \mathrm{GeV}$ are selected for further analysis, as shown in Fig. 2.

A binned maximum likelihood fit to the invariant mass distribution in Fig. 2 is applied to extract the number of $B^{ \pm}$mesons. The background processes considered are $B^{ \pm} \rightarrow J / \psi \pi^{ \pm}$, where the kaon mass is wrongly assigned to the pion, $B^{ \pm / 0} \rightarrow J / \psi K^{* \pm / 0} \rightarrow J / \psi K \pi$ and $B^{ \pm / 0} \rightarrow$ $J / \psi K^{ \pm} \pi^{0 \mp}$, where the final state pion is not associated to the decay vertex, and combinatorial background from random combinations of $J / \psi$ with a track. The total number

\footnotetext{
${ }^{2} B^{+}$refers to either of the two charged states, under the assumption that the two production cross sections are equal
}

of signal $B^{ \pm}$events observed in the full $p_{T}$ and $y$ range is $125600 \pm 1201$, where the uncertainty is statistical.

The differential cross section for $B^{+}$meson production is measured using:

$$
\frac{d^{2} \sigma\left(p p \rightarrow B^{+} X\right)}{d p_{T} d y}=\frac{N_{B^{+}}}{\int \mathcal{L} d t \times \mathcal{B} \times \Delta p_{T} \times \Delta|y|}
$$

where $\mathcal{B}$ is the branching ratio of the signal decay, $\mathcal{B}\left(B^{+} \rightarrow\right.$ $\left.J / \psi K^{+} \rightarrow \mu^{+} \mu^{-} K^{+}\right)=(6.03 \pm 0.21) \times 10^{-5}$. Efficiency and acceptance corrections are applied to correct for trigger, geometry and detector effects.

The differential cross section of $B^{+}$production is shown in Fig. 2, as a function of $p_{T}$ for different $|y|$ ranges. The results are compared with NLO theoretical predictions from POWHEG [7] and MC@NLO [8]. Within the large theoretical uncertainties $(\approx 20-40 \%)$, the POWHEG predictions agree with the measurement in absolute scale and in the dependencies on $p_{T}$ and $y$. At low y, MC@NLO+Herwig a lower production cross section than that observed in the data, and exhibits a softer $p_{T}$ spectrum, which becomes harder for higher $y$.

\section{$5 W+J / \psi$ associated production}

The associated production of a $J / \psi$ meson and a $W$ boson provides a powerful probe of the production mechanism of charmonium in hadronic collisions. In addition to the single-parton scattering (SPS) process, double-parton scattering (DPS) interactions [9], where two pairs of partons create an uncorrelated $J / \psi$ and $W$, give rise to a signature which is indistinguishable from true associated production (SPS) on an event-by-event basis. The first observation of $W^{ \pm}+$prompt $J / \psi$ associated production is presented in the $W^{ \pm} \rightarrow \mu^{ \pm} v$, and $J / \psi \rightarrow \mu^{+} \mu^{-}$channel. The cross section ratio of $W^{ \pm}+$prompt $J / \psi$ to inclusive $W^{ \pm}$production is also measured, along with an estimate of the DPS contribution.

Using $4.6 \mathrm{fb}^{-1}$ of data, events are pre-selected with a high- $p_{T}$ muon trigger. The trigger muon is required to match a reconstructed muon with $p_{T}>25 \mathrm{GeV}$ and $|\eta|<2.4$. Two additional oppositely-charged muons with $p_{T}>2.5 \mathrm{GeV}$ and $|\eta|<2.5$, of which at least one has $p_{T}>4 \mathrm{GeV}$, are required in the event to reconstruct the $J / \psi$ candidate. The invariant mass of the dimuon system is required to be within $2.5 \mathrm{GeV}<m_{\mu \mu}<3.5 \mathrm{GeV}$. The transverse energy-momentum imbalance measured in the detector, $E_{T}^{\text {miss }}$, must exceed $20 \mathrm{GeV}$, and the $W$ boson transverse mass $m_{T}(W)^{3}$ must exceed $40 \mathrm{GeV}$. Events with invariant mass of two oppositely charged muons within $10 \mathrm{GeV}$ of the $Z$ boson mass are rejected. Events with $J / \psi p_{T}$ in $8.5 \mathrm{GeV}<J / \psi p_{T}<30 \mathrm{GeV}$ are kept for further analysis.

A two-dimensional maximum likelihood fit on the dimuon invariant mass and $J / \psi$ pseudo-proper time is used to separate the $J / \psi$ from the combinatoric background and the prompt $J / \psi$ signal from the non-prompt $J / \psi$ background, as shown in Fig. 3. This is followed by a fit to

$$
{ }^{3} m_{T}(W) \equiv \sqrt{2 p_{T}(\mu) E_{T}^{\mathrm{miss}}\left(1-\cos \left(\phi^{\mu}-\phi^{v}\right)\right)}
$$



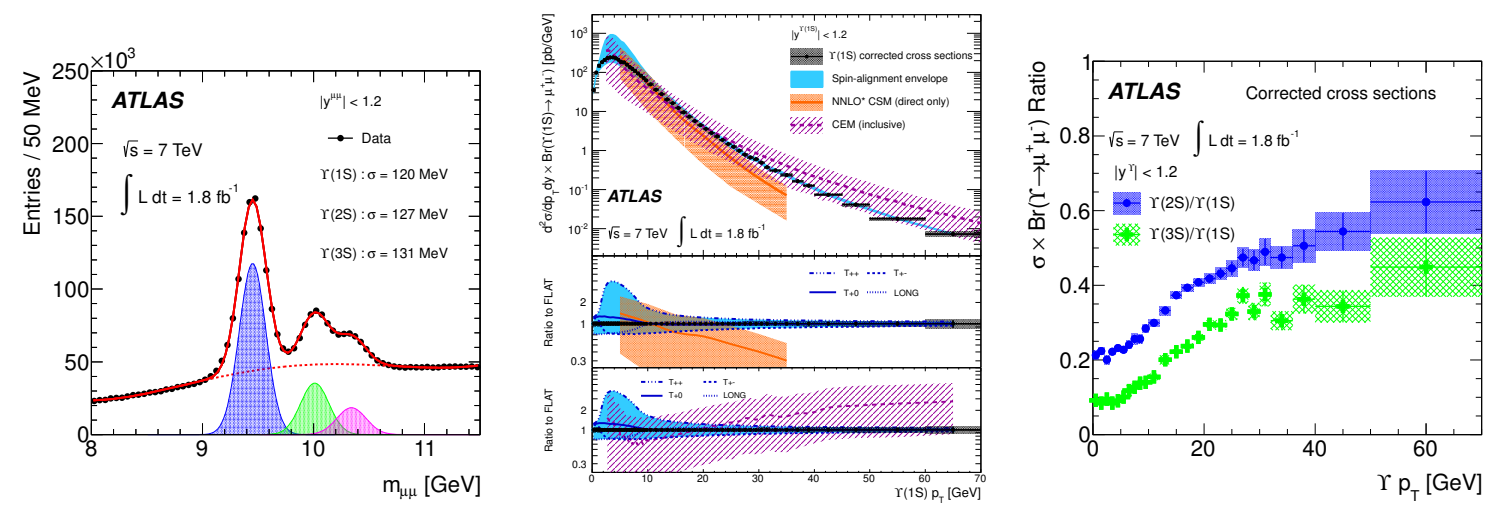

Figure 1. The di-muon candidate events with the fit to extract the number of $\Upsilon(1 \mathrm{~S}, 2 \mathrm{~S}, 3 \mathrm{~S})$ for the central region (left). The differential cross section for $\Upsilon(1 \mathrm{~S})$ candidates as a function of transverse momentum in the central region (center). Theoretical predictions from the color singlet (CSM) [5] and color evaporation (CEM) [6] models are superimposed. The ratios $\Upsilon(2 S): \Upsilon(1 S)$ and $\Upsilon(3 S): \Upsilon(1 S)$ as functions of transverse momentum (right). Figures are from [2].
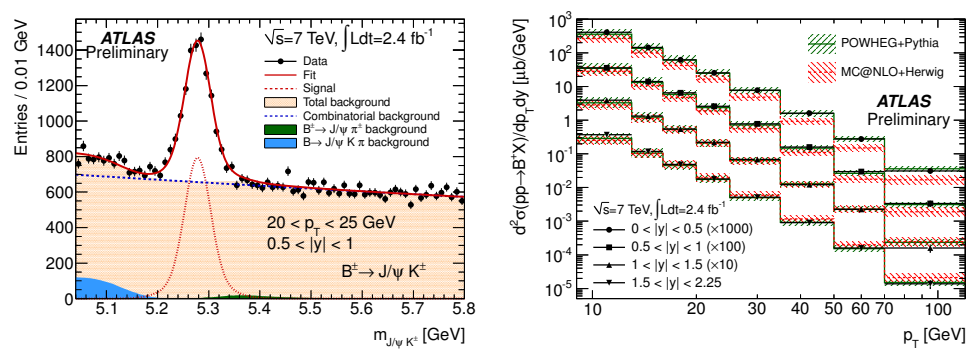

Figure 2. The binned maximum likelihood fit to the invariant mass distribution of the $B^{ \pm}$candidates in a specific $p_{T}$, $|y|$ region, which is used to extract the number of $B^{ \pm}$mesons in the data (left). The differential cross section of $B^{+}$production as a function of $p_{T}$ for different $|y|$ ranges (right). The results are compared with NLO predictions from POWHEG and MC@NLO. Figures are from [3].

the $m_{T}(W)$ distribution to determine the contributions of $W^{ \pm}$and multijet background produced in association with a prompt $J / \psi$.

The number of $W+$ prompt $J / \psi$ events is $29.2_{-6.5}^{+7.5}$, observed with a statistical significance of $5.3 \sigma$. The contribution of DPS interactions is estimated to be $10.8 \pm 4.2$ events, using the measured $J / \psi$ cross section [10] and the effective cross section of DPS $W+2$ jets events [11] with ATLAS. Similarly, the expected contribution from pileup interactions is estimated to be $1.8 \pm 0.2$ events, while the multijet background is $0.1 \pm 4.6$ events, as estimated from the $M_{T}(W)$ fit. The azimuthal angular separation between the $W$ boson and the $J / \psi$ meson is expected to be uniform for DPS production, and peaked at $\pi$ for SPS, and the observed distribution indicates the presence of both contributions, as shown in Fig. 3.

The fiducial cross section ratio of the $W+$ prompt $J / \psi$ production to inclusive $W$ production, $R^{\text {fid }}=(50 \pm 12 \pm 4) \times$ $10^{-8}$, is measured after efficiency corrections to account for muon reconstruction. After applying acceptance corrections which depend on the $J / \psi$ spin-alignment, the inclusive cross section ratio, $R^{\text {incl }}=\left(123 \pm 31 \pm 10_{-24}^{+40}\right) \times 10^{-8}$, is measured. Subtracting the estimated DPS contribution yields the DPS-subtracted ratio, $R^{\text {DPS sub }}=(74 \pm 31 \pm$ $\left.21_{-24}^{+40}\right) \times 10^{-8}$, which can be compared with theoretical expectations for SPS production in the color singlet [12] and color octet [13] models, as shown in Fig. 3. Large statistical uncertainties imply that the current predictions are compatible with the measurement at the $2 \sigma$ level.

\section{Conclusions}

ATLAS has a rich heavy flavor and quarkonia program with measurements and searches that can contribute to a better understanding of Quantum Chromodynamics. Using data from proton-proton collisions in the LHC at $\sqrt{s}=$ $7 \mathrm{TeV}$ the $\Upsilon(1 \mathrm{~S}, 2 \mathrm{~S}, 3 \mathrm{~S})$ production cross sections and the charged $B$ meson production cross section measurements are presented, along with comparisons with theoretical expectations. The observation of $J / \psi$ produced in association with $W$ bosons, accessible for the first time, can provide new insights into the hadro-production of charmonium.

\section{Acknowledgements}

The author was supported by the US National Science Foundation, the University of Chicago and the Onassis Scholarships Foundation.

\section{References}

[1] ATLAS Collaboration, JINST 3, S08003 (2008) 

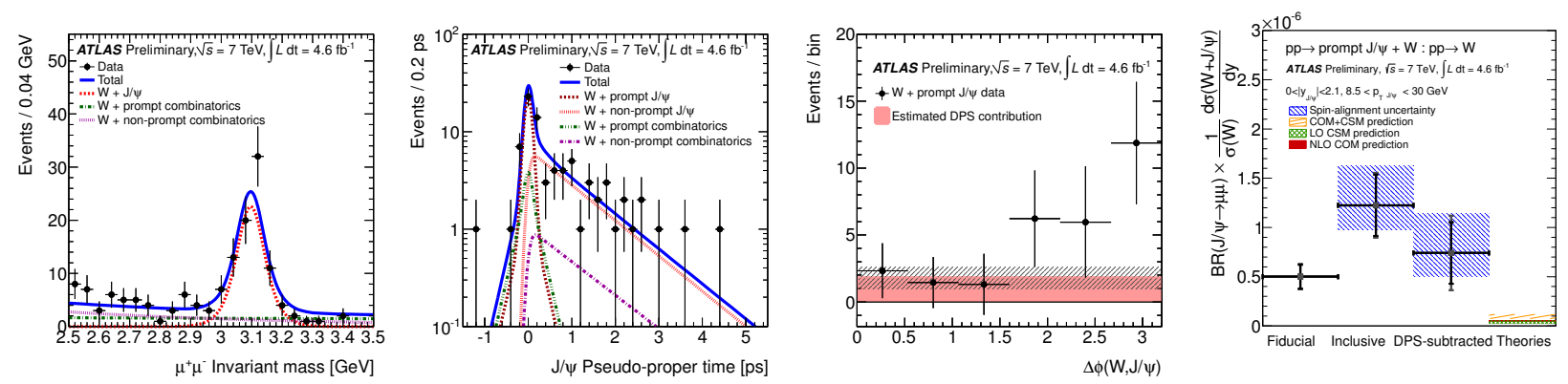

Figure 3. The two-dimensional maximum likelihood fit on dimuon invariant mass (left) and $J / \psi$ pseudo-proper time (center-left) used to extract the prompt $J / \psi$ component. The azimuthal angle between the $J / \psi$ and $W$ indicates the presence of both SPS and DPS production (center-right). The fiducial, inclusive and DPS-subtracted $W+$ prompt $J / \psi: W$ ratios are shown, as well as theoretical expectations from color singlet and color octet models (right). Figures are from [4].

[2] ATLAS Collaboration, Phys. Rev. D 87, 052004 (2013)

[3] ATLAS Collaboration, ATLAS-CONF-2013-008 (2013)

[4] ATLAS Collaboration, ATLAS-CONF-2013-042 (2013)

[5] P. Artoisenet, Phys. Rev. Lett. 101, 152001 (2008);

J.P. Lansberg, Eur. Phys. J. C 61, 693 (2009);

[6] A.D. Frawley, T. Ullrich and R. Vogt, Phys. Rep. 462, 125 (2008)

[7] P. Nason, JHEP 0411, 040 (2004); S. Frixione, P. Nason and G. Ridolfi, JHEP 0709, 126 (2007)
[8] S. Frixione, JHEP 0206, 029 (2002); S. Frixione, JHEP 0308, 007 (2003)

[9] N. Paver and D. Treleani, Nuovo Cim. A70, 215 (1982)

[10] ATLAS Collaboration, Nucl. Phys. B850, 387 (2011)

[11] ATLAS Collaboration, New J. Phys. 15, 033038 (2013)

[12] J.P. Lansberg, arXiv:1303.5327 [hep-ph] (2013)

[13] K.T. Chao, Phys. Rev. Lett. 108, 242004 (2012) 